\title{
SSinteza
}

Impact of Internet on Business Activities in Serbia and Worldwide

Uticaj Interneta na poslovanje u Srbiji i svetu

DOI: 10.15308/SINTEZA-2014-62-65

\section{THE EPISTEMOLOGY OF MATHEMATICS IN THE DIGITAL AGE}

\author{
Snezana Lawrence \\ Bath Spa University, UK
}

\begin{abstract}
:
This paper attempts to define some of the questions that are emerging in the digital era of learning and communicating mathematics. The paper explores the epistemological questions and problems in the discipline of mathematics education in the digital age, comparing it to the examples from the past when new technologies changed the nature of the learning of mathematics in as drastic way as the Internet did to us today.
\end{abstract}

\section{Key words:}

digital, era,

epistemology,

mathematics,

Internet,

communication.

\section{SETTING THE SCENE}

This paper attempts to define some of the questions that are emerging in the new digital era of learning and communicating mathematics. The paper is therefore first exploratory: it defines and explores the new epistemological questions and problems in the discipline of mathematics education as they arise with the ever increasing use of the Internet and its ability to spread information of all kinds, including that about mathematics. Secondly, the paper is speculatory: whilst it speculates on the issues that arise from the changes of environment, culture, and even nature in the learning of mathematics, it does not offer much empirical evidence - perhaps this is something that will be undertaken following the present study. Thirdly, this paper is a general study of the use of Internet based information that can be used for the learning of mathematics, and is not bound to any specific country or culture, but is heavily biased towards English-speaking resources as the author is British-based and all the resources of mathematics that are discussed are English-language based. This latest statement would need further exploration and investigation itself: is English really, as popularly believed, currently the international language of education and how does the learning of mathematics depend on the language and culture hence associated with it? We will leave this particular question aside and pay attention to it in another communication.

\section{THE CHANGES IN THE EPISTEMOLOGY OF MATHEMATICS}

\section{Learning Mathematics up to 2000}

The epistemology of mathematics is a large field of en- the ways we learn about mathematics, and usually gives views of how this is done: throughout the history various social groups, including those growing out of or belonging to the state apparatus, used epistemology of mathematics as a way of justifying and organizing their systems of learning mathematics. Until recently, the process was of a mainly one-way process and it went in this direction: the mathematics was created by mathematicians, the philosophers of mathematics and mathematics educationalists studied how this is done, and their work was interpreted (rarely successfully) into systems which could be adopted for schools (see figure 1 below). The education system then interpreted what they were told the curriculum is or should be, and fed the diet of what was regarded as nutritious mathematics to the armies of young people. Depending on the quality of teachers and their ability to make sense of twice or three-times watered-down versions of mathematics and how it should be done, children were able to make sense for themselves of mathematics and how to do it. The publishing industry sometimes stepped in between and added another layer of interprentation, but the direction was still the same - towards the learners and without much possibility for any further interaction in the opposite direction.

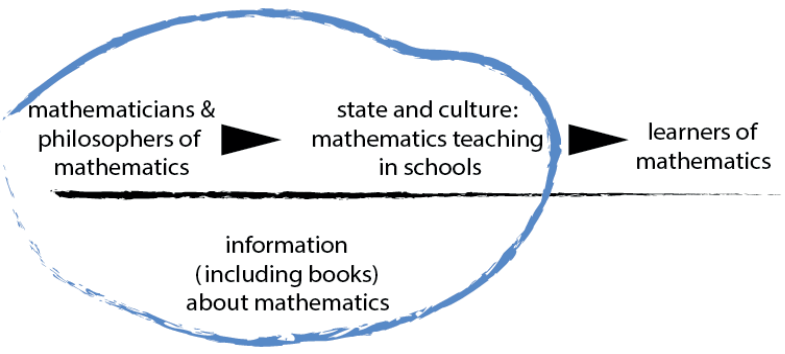

The mainly one-way relationship of directional instruction about what mathematics is, or was, prevalent until around 2000. 
Learning Mathematics since the 2000

The modern technology increasingly interferes with this model of mathematics education. In between mathematics and mathematicians there is increasingly less of state and education apparatus or publishing industry's interference and interpretation for the benefit of the learners of mathematics. What is on the Internet now is a mixture of paid-for and free content, scholarly-created and amateur-written, collaborative or lone-internet-ranger content. And so the learner, of whatever age, is able to, with enough effort, make sense of mathematics, what it is, and how to do it, by themselves.

Equally, the 'state and culture' category from figure 1 is increasingly not the official state or the culture defined by the state or nation, but more fluid and still to be defined system of the new emerging groups of interpreters of knowledge. Who then defines what mathematics should be learnt? And who is teaching it? Let us leave aside for the moment the question of who is teaching this mathematics, and look at what mathematics is there to be learnt in this new Brave New Digital World.

I have identified three 'sets' of mathematics 'curricula' which are getting more clearly defined today and are offered to the Internet users:

- The various curricula and courses for the learning of mathematics; perhaps the most influential of all are the curricula for the university entrance examinations. In this category fall also the content of mathematics qualifications that one needs in order to gain access to further education and training. This set is defined by the examination bodies, the universities, and increasingly by industry. This set is still clearly defined by national boundaries.

- Websites giving some kind of explanation or instruction in mathematics - these range from simple text sites to sophisticated online paying or subscription sites and free courses by the leading schools of mathematics from around the world. This is the fastest growing set of learning resources in mathematics but one that does not provide a feedback to the learner.

- The growing digital tools which are upgraded and updated constantly such as apps and online subscription sites, which give immediate feedback and learning/improvement tools. These resources are almost exclusively produced on a business model basis.

The development of these digital resources for the learning of mathematics changed the learning environment in mathematics in several aspects, although it has not had the same effect on the mathematics education sufficiently enough. This means that, whilst the national reports on mathematics education still record the low engagement with the subject, and school mathematics is often considered 'boring and irrelevant', the curiosity about mathematics has improved and the popular interest in mathematics increased to such a degree that mathematics is, perhaps for the first time since the Renaissance, considered again to be an exciting and mysterious field of study worthy of attention of not only geeks but the most talented of us.

What are the implications of this change? I will mention several such important instances.

The illustration below serves well to develop this argument:

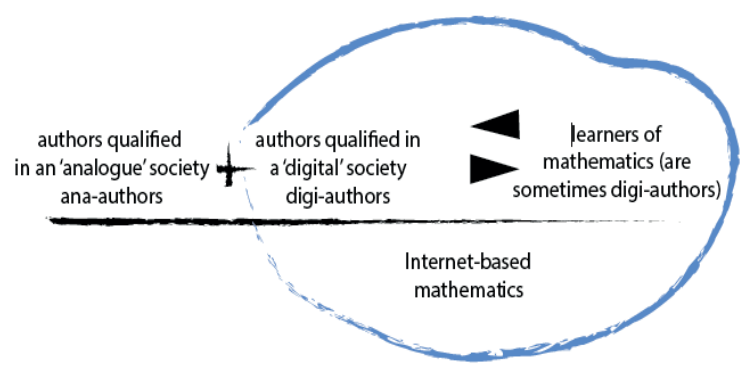

The many-to-many directional relationship of learning, rather than the instruction in mathematics, is a model emerging since c. 2000.

\section{THE NEW (BRAVE) MODEL OF LEARNING}

In this new model the learners are at the same time authors of instructional materials, something that, although it seems very new, has been present in the past too. Two generic examples come to mind - the times when the new communication technique is invented and becomes accessible to a larger number of people than before: the invention of the printing press is one such example. A proliferation of mathematics textbooks in the 16th century, including the edition of Euclid's Elements by Billingsley, (and prefaced by Dee) in 1570 in England revolutionized the learning of mathematics by the simple use of English rather than Latin language. Publishing books on mathematics in the vernacular changed the audience from students and scholars at the old universities to anyone who could afford to buy or borrow the books on the subject. ${ }^{1}$ Equally, the similar 'revolutions' in the learning of mathematics happened with the emergence of new professions: the 18th and 19th centuries saw the rise and the proliferation of textbooks on practical mathematics for the newly emerging engineering and architectural professions. ${ }^{2}$

\section{The learning establishments}

These two broadly described examples: the invention of the printing press and what it meant to the learning of mathematics, and the emergence of new professions that needed high content of mathematical knowledge and what that meant to the learning of mathematics, both have elements of the changes that are taking place today in mathematics education.

Firstly, the learning establishments are affected themselves. Just as Oxford and Cambridge had to open up to the learning in vernacular language in the 16th century and eventually the new universities were founded to accommodate not only this but other 'leveling' aspects of

1 See for example Lawrence [4].

2 See Lawrence [5]. 
class and religious divisions in the 19th century, so too the great universities of today are opening up to a wider public. This means that the learners who may not have the usual prerequisite qualifications usually needed to enter such universities are being offered a lot of free online content from these univerisites via the Internet. ${ }^{3}$

Secondly, those who professionally profited in the periods of two paradigm changes described above were not professional mathematicians themselves: they were in fact interested in mathematics from the very edges of what we could identify as professional circle of mathematics scholars. Their interest in the subject, and their ability to describe mathematics in popular and easily accessible way made their publications used widely. Today we also have authors who are themselves learners of mathematics. Most recently, for example, I have noticed that people use 'blogger' as a term to describe their professional identity surprising perhaps to those of us who haven't got millions of followers and therefore companies keen to advertise on our blogs. The boundaries are currently blurred between those who know and don't necessarily know much - everyone is learning something, be it how to update the information on a wiki page which is wrong or what is wrong about the maths on that wiki page.

The type of the learners of mathematics is inevitably changing also. The ways of learning mathematics are diversifying and both the need for qualifications in mathematics and their relevance in the world are undergoing major overhaul. ${ }^{4}$ The reasons for the learning of mathematics are shifting: the question "why do we need to know this?" which mathematics teachers experience on a daily basis is replaced by the greater awareness why you actually do perhaps need to know some mathematics in order to participate in the invention of new technologies, including the development of Internet-based tools and services.

\section{HOW DO WE KNOW WE KNOW IT?}

Does this influence the actual development of the epistemology of mathematics in the digital age? The questions such as "what are the origins of mathematical knowledge" and "how does the knowledge of mathematics develop?" will inevitably undergo some changes also but how can we predict in which direction? Until now, the learning of mathematics was measured via state channels of varying kind, from national to international bodies, and the tests such as OECD or Pisa surveys showed how well countries were doing in mathematics education. But as the tools for the learning, the learning environment itself, and the purposes of learning mathematics change, so does the nature of these questions. We may now perhaps begin to ask ourselves: "who invented mathematics?"5 or do a survey of a kind - "mathematics - frequently asked questions".

3 Various versions of MOOC (Massive Open Online Course) are being currently developed: the online courses aimed at unlimited participation of learners via the Internet.

4 See Lawrence

5 Facebook group on "who invented mathematics" has a growing number of members and is maintained by the British Society for the

\section{What do we know of mathematics via the Internet?}

Is mathematics an instrument or a discipline, product of an objective absolute truth or is it built through the context from which it arises, by mathematicians who, as all humans, work from within their own constraints of existence? ${ }^{6}$ These are perhaps the two views which are opposite in their attempt to define mathematics, and if one accepts the absolutist (former) stance then one will find this paper somewhat superfluous: mathematics is and how it becomes, in what order and in what way, is irrelevant. But majority of mathematicians, mathematics philosophers and mathematics educators, accept that there is some degree to which mathematics is subject to the context from which it becomes and that what comes out, where, in what time and by whom is what defines mathematics itself. This communication is far too short to go deeper into the questions that can be posed around this issue, but consider then that most of the learning of mathematics these days is done on-line. Learners learn about what mathematics is, who mathematicians are, how mathematics became to be a discipline, who invented what, what various concepts mean, and the order in which they were invented, through the internet. There is a new hierarchy being born of the concepts of mathematics and it is done by Google ranking.

\section{How will we in the future start measuring the success of learning mathematics?}

How will we know whether mathematics that people think they know and the mathematics they actually know are more or less the same? Some politicians for example seem to believe that the success of industrial development is directly linked to the success in the learning of mathematics: current programme whereby Chinese teachers are invited into English classrooms is just one such example. ${ }^{7}$ In our previous official systems, where there was a direct link between what is learned to what is examined it should be pretty much clear what needs to change in order to get better results. However, in a system where the learners are creators of knowledge and perpetuators of generating new knowledge, the cycle of learning is more complex. This is now under complete flux and therefore the governments and teachers are wondering what they can do to improve - but perhaps they should look instead more closely at what is going on in the digital world.

6 Burton in [2] constructs a framework for an epistemological model to describe the links between coming to know mathematics, as a researcher, and as a learner. The five categories Burton gives are: - person- and cultural-social relatedness; aesthetics; intution and insight; styles of thinking; connectivities.

7 The 2014 initiative by the British Government to invite Chinese teachers into Mathematics Classrooms around the country to pass on some of their successes to their British colleagues in how to teach for success in mathematics. See for example http://www.thetimes. co.uk/tto/education/article4030303.ece 


\section{THE EVER-GROWING GIMMICK MATH(S)}

We face some real difficulties in the future and some dangers that we previously did not know of - in particular what I call gimmick math. I see as one of the greatest challenges for the future of learning of mathematics the uncertainty of the ways we measure the ability to actually do mathematics and the perceived ability of an individual. In the traditional model of mathematics education, this was tested in various ways and the go-ahead to the learner to progress further and apply knowledge was carefully negotiated. In the digital age this has changed. The sites that depend on the amount of internet traffic purposefully post questions or offer insight into mathematics via some fact that your maths teacher hid from you. These are usually 'trick' questions or gimmick facts and have little to do with actual mathematical thinking. I give one such illustration of here:

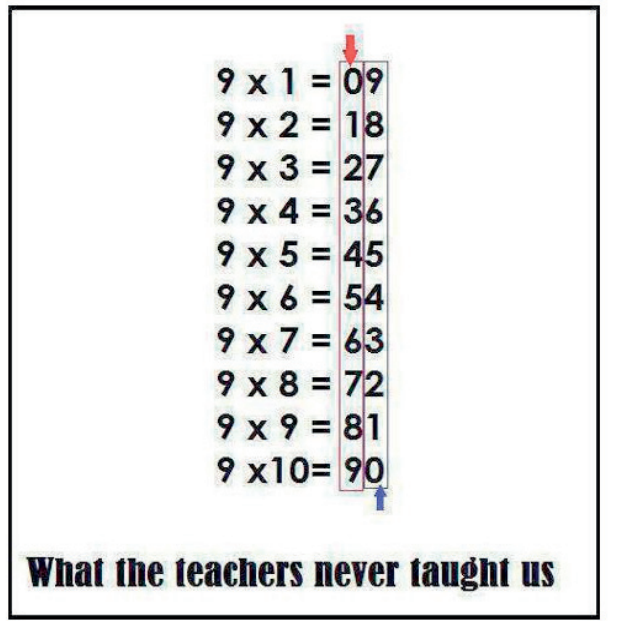

Apparently useful and quick way of doing a nine times table - something maths teachers don't seem to teach...

The result is what I called 'gimmick math': not really mathematics but a pretty-random collection of poorly constructed mathematical facts professing to be clever. Sometimes, as in the example above (figure 3 ), this is given with an additional spice of rebellion against the established routines of mathematics education.

What can the outcome be? Any number of horrid things: we can have a generation of people who know some silly tricks which are nothing to do with mathematics but call themselves mathematicians; we can have people who think they actually know mathematics and are put in charge of any number of things - electric grid supply, flight control, national statistics... counting election ballots.

\section{CONCLUSION}

What are then the conclusions of this short communication? The first one would be that the difference between the way we learn mathematics has I believe, changed for ever by the invention of the Internet: this is now the allinclusive way to generate communications and the teachers and learners are interchangable groups of people. The questions is still who knows maths, and how do you measure the mathematics knowledge. That will be a chal- lenge of the new digital world. The motivation to learn the subject however, seems to be changing towards the everincreasing positive attitudes in popular culture. Not only are mathematicians becoming intersting, but they can also be deadly sexy beasts such as Larsson's Girl. ${ }^{8}$

\section{ACKNOWLEDGMENT}

I am indebted to Brendan Larvor from the University of Hertfordshire, England, for organising three conferences in London on Mathematical Cultures, 2012-2014, which made me think about and articulate and hence record most of the content of this paper. These conferences made me reflect on the importance of the cultural context of learning and doing mathematics.

\section{REFERENCES}

[1] B. Buldt, B. Löwe, and T. Müller, "Towards a New Epistemology of Mathematics" in Erkenntnis (1975-), Vol. 68, No. 3, p;p. 309-329. 2008.

[2] L. Burton, "Mathematics and Their Epistemologies - and the Learning of Mathematics". European Research in Mathematics Education I: Group 1, 1999.

[3] W. Dörfler, "Mathematics and Mathematics Education: Content and People, Relation and Difference", in Educational Studies in Mathematics, Vol 54, No. 2/3, Connecting Research, Practice and Theory in the Development and Study of Mathematics Education, 147-170, 2003.

[4] S. Lawrence, "Dee and his books: lessons from the history of mathematics for primary and middle school teachers", BSHM Bulletin: Journal of the British Society for the History of Mathematics, Vol. 26, No. 3, pp. 160-166, 2011.

[5] S. Lawrence, Geometry of Architecture and Freemasonry in $19^{\text {th }}$ century England, PhD Thesis, Open University England, 2002.

[6] S. Lawrence, "Inquiry based mathematics teaching and the history of matheamtics in the English curriculum", Proceedings of the $6^{\text {th }}$ European Summer School in the History and Philosophy of Mathematics, Vienna, July 2010.

[7] S. Lerman, "Alternative Perspectives on the Nature of Mathematics and Their Influence on the Teaching of Mathematics", British Educational Research Journal, Vol. 16, No. 1, pp. 53-61, 1990.

[8] R. Noss, "For a Learnable Mathematics in the Digital Culture", Educational Studies in Mathematics, Vol. 48, No. 1, pp. 21-46, 2001.

[9] A. Sierpinska and S. Lerman "Epistemologies of Mathematics and of Mathematics Education" in A. J. Bishop et al. (eds.) International Handbook of Mathematics Education, pp. 827-876. Kluwer Academic Publishers, Netherlands.

[10] D. L. Wheeler and D. Montgomery, "Community College Students' Views on Learning Mathematics in Terms of Their Epistemological Beliefs: A Q Method Study" in Educational Studies in Mathematics, Vol. 72, No. 3, pp. 289306, 2009.

8 The Girl Who Played with Fire is Stieg Larsson's novel (or one in a series) in which the main role is given to a girl who is madly interested in mathematics and Fermat's Last Theorem. Her name is Salander. 